\title{
MODELOS DE ORIENTACIÓN E INTERVENCIÓN PSICOPEDAGÓGICA: MODELO DE INTERVENCIÓN POR SERVICIOS ${ }^{1}$
}

\author{
Esperanza Bausela Herreras
}

Universidad de León

\section{CONCEPTUALIZACIÓN DE MODELO DE ORIENTACIÓN}

La orientación como cualquier disciplina de acción, cuenta con una serie de modelos de intervención que suponen distintos modelos de organización y que ofrecen distintas posibilidades de acción. Estos modelos nos servirán como marcos de referencia a la hora de diseñar planes de actuación (Castellano, 1995).

Vamos a referirnos a los modelos de intervención como estrategias para conseguir unos resultados propuestos. Los modelos de orientación según Bisquerra (1998) sugieren procesos y procedimientos concretos de actuación, es decir, se pueden considerar como "guías para la acción". A lo largo de la historia han ido surgiendo diversos modelos. La investigación ha contribuido en determinar la eficiencia de estos modelos.

En el marco de la orientación educativa encontramos, por ejemplo, tres definiciones especificas:

- "Son estrategias fundamentadas que sirven de guía en el desarrollo del proceso de Orientación en su conjunto (planificación, puesta en práctica y evaluación) o en alguna de sus fases..."(Bisquerra, 1992: 177).

- "La representación de la realidad sobre la que hay que intervenir, y que va a influir en los propósitos, los métodos y los agentes de dicha intervención" (Rodríguez Espinar y otros, 1993).

- "Son una representación que refleja el diseño, la estructura y los componentes esenciales de un proceso de intervención" (Álvarez y Bisquerra, 1997: 23).

Como ha señalado Rodríguez Diéguez (1990:56) en Orientación "los modelos están desempeñado la función de hacer más accesibles las construcciones teóricas mediante aproximaciones sistemáticas, así como la de seleccionar aquellos hechos de la realidad que, sometidos a investigación, puedan contribuir a la elaboración de teorías".

\section{TIPOLOGÍAS DE MODELOS DE ORIENTACIÓN}

Bisquerra y Álvarez (1996) han señalado recientemente que todavía es escasa la literatura sobre modelos de orientación y sus tipos, no obstante diversos autores han realizado propuestas de clasificación de los modelos de intervención en orientación.

\footnotetext{
${ }^{1}$ Este estudio se enmarca dentro del desarrollo de la tesis doctoral "Diseño y Desarrollo de un Servicio de Orientación y Asesoramiento psicológico a la diversidad en el contexto de la Universidad de León”, codirigida por Delio del Rincón Igea y Dionisio Manga Rodríguez de la Universidad de León.
} 
Es posible encontrar distintas clasificaciones de modelos según el criterio utilizado. Así, Velaz y Ureta (1998) recogen la siguiente clasificación:

(a) Empleando un criterio histórico, Rodríguez Monereo (1995:21 - 51), distingue los siguientes "modelos de orientación educativa y profesional en el siglo XX":

Modelos históricos; el modelo de Orientación Vocacional de Frank Parsons (1980), y el modelo de Brewer que asimilaba la orientación y la educación (1914).

Modelos modernos de Orientación Educativa y profesional;

- La orientación entendida como clasificación y ayuda al ajuste o adaptación (de Koos y Kefauver, 1932).

- La orientación como proceso clínico.

- La orientación como consejo proceso de ayuda para la toma de decisiones.

- La orientación como sistema metodológica ecléctico

Modelos contemporáneos de Orientación (centrados en la institución escolar y en las organizaciones educativas);

- La orientación como un conjunto o constelación de servicios.

- La orientación como reconstrucción social.

- La orientación como acción intencional y diferenciada de la educación.

- La orientación facilitadora del desarrollo personal.

- Modelos centrados en las necesidades sociales contemporáneas;

- La orientación como técnica consultiva o intervención indirecta.

- Las intervenciones primarias y secundarias: la teoría de la orientación activadora.

- Los Programas Integrales de Orientación preventiva.

- Orientación para la adquisición de las habilidades de vida.

(b) Parker (1868), clasifica los modelos en función del estilo y la actitud del orientador en el desarrollo de su función, en relación con dos ejes (directividad - no directividad y enfoque existencialista conductista), distinguiendo; modelo de rasgos y factores, modelo espontáneo - intuitivo; modelo rogeriano; modelo pragmático - empírico; modelo conductista; modelo ecléctico.

(c) En función del tipo de relación que se establece entre orientador y orientado, Escudero (1986) clasifica los modelos del siguiente modo;

a. Modelo psicométrico: el orientador es el experto de una serie de técnicas y el profesor el destinatario de los resultados de las mismas.

b. Modelo clínico - médico; basada en el diagnóstico. El orientador diagnóstico y diseña el plan de intervención, que es aplicado pasivamente por el profesor. 
c. Modelo humanista; la orientación es un proceso de ayuda al individuo en un clima positivo de relación. El profesor es concebido como orientador.

Santana Vega y Santana Bonilla (1998:69) proponen la siguiente clasificación de modelos de intervención en orientación (figura 1):

\begin{tabular}{|c|c|}
\hline Autores & $\begin{array}{l}\text { Clasificación de modelos de } \\
\text { intervención en orientación }\end{array}$ \\
\hline $\begin{array}{l}\text { Rodríguez Espinar (1993) } \\
\text { Álvarez González (1995) }\end{array}$ & $\begin{array}{l}\text { Modelo de intervención directa } \\
\text { individual (modelo de counseling). } \\
\text { - Modelo de intervención grupal } \\
\text { (modelo de servicios vs programas). } \\
\text { - Modelo de intervención indirecta } \\
\text { individual y/o grupal (modelo de } \\
\text { consulta). } \\
\text { - Modelo tecnológico. }\end{array}$ \\
\hline Álvarez Rojo (1994) & $\begin{array}{l}\text { Modelo de servicios. } \\
\text { - Modelo de programas. } \\
\text { problemas educativos. } \\
\text { - Modelo de consulta centrado en las } \\
\text { organizaciones. }\end{array}$ \\
\hline Bisquerra y Álvarez (1996) & $\begin{array}{l}\text { - } \quad \text { Modelo clínico. } \\
\text { - } \quad \text { Modelo de servicios. } \\
\text { - } \quad \text { Modelo de de consulta. } \\
\text { - } \quad \text { Modelo tecnológico. } \\
\text { - }\end{array}$ \\
\hline Repetto (1995) & $\begin{array}{l}\text { - } \quad \text { Modelo de consejo (counseling). } \\
\text { - } \quad \text { Modelo de servicios. } \\
\text { - } \quad \text { Modelo de conramalto tecnológico. } \\
\text {. }\end{array}$ \\
\hline $\begin{array}{c}\text { Jiménez Gómez y Porras Vallejo } \\
\text { (1997) }\end{array}$ & $\begin{array}{l}\text { Modelo de counseling (acción } \\
\text { psicopedagógica directa individualizada) } \\
\text { psicopedagógica directa grupalon } \\
\text { psicán } \\
\text { psicopedagóde consulta (acción } \\
\text { grupal). }\end{array}$ \\
\hline
\end{tabular}

Figura 1 Clasificación de modelos de intervención en orientación (tomado de Santana Vega y Santana Bonilla, 1998:69).

Castellano (1995) diferencia los siguientes modelos:

- Modelo de counseling; se centra en la acción directa sobre el individuo para remediar situaciones déficit. Aunque desligado del proceso educativo, su demanda aconseja una prudente utilización.

- Modelo de consulta; centrado en la acción indirecta sobre grupos o individuos, ha adquirido un gran auge, ejerciendo su función desde una perspectiva terapéutica, preventiva o de desarrollo.

- Modelo tecnológico; con las limitaciones propias de cualquier medio tecnológico y el desconocimiento del mismo, este modelo, que pretende fundamentalmente informar y que goza de un importante auge en otros países, desarrolla en el nuestro experiencias por el momento poco relevantes. 
- Modelo de servicios; se centra en la acción directa sobre algunos miembros de la población, generalmente en situaciones de riesgo o déficit.

- Modelo de programas; una intervención a través de este modelo de acción directa sobre grupos, es una garantía del carácter educativo de la orientación.

- Modelo de servicios actuando por programas; Este nuevo modelo de intervención directa sobre grupos, presenta la particularidad de considerar el análisis de necesidades como paso previo a cualquier planificación, y una vez detectadas y priorizada dichas necesidades, diseñar programas de intervención que den satisfacción a las mismas.

En un trabajo desarrollado por Repetto (1994) con objeto de valorar los logros de las investigaciones básicas y aplicadas en la Orientación Educativa entendida como Intervención Psicopedagógica, diferencia cuatro tipos de modelos; Asesoramiento o Consejo (counseling), servicios puros o mixtos, programas, consulta y tecnológico, centrándose en su estudio se centra en el modelo tradicional de carácter terapéutico y personalizado que reside en el Asesoramiento o Consejo y en el modelo de intervención por programas.

Álvarez y Bisquerra (1997) clasifican los modelos de orientación combinando tres criterios no excluyentes (ver figura 2); (i) El carácter teórico del modelo, (ii) el tipo de intervención y (iii) el tipo organización o institución en la que se lleva a cabo.

\begin{tabular}{|c|c|c|}
\hline \multicolumn{3}{|c|}{ TEÓRICOS } \\
\hline \multirow[t]{2}{*}{$\begin{array}{l}\text { MODELOS DE } \\
\text { INTERVENCIÓN }\end{array}$} & BÁSICOS & $\begin{array}{l}\text { - Clínico } \\
\text { - Programas } \\
\text { - Consulta } \\
\end{array}$ \\
\hline & MIXTOS & $\begin{array}{l}\text { - El modelo psicopedagó- } \\
\text { gicos }\end{array}$ \\
\hline \multirow[t]{2}{*}{$\begin{array}{c}\text { MODELOS } \\
\text { ORGANIZATIVOS }\end{array}$} & $\begin{array}{c}\text { MODELOS } \\
\text { INSTITUCIONALES }\end{array}$ & $\begin{array}{l}\text { - M.E.C. } \\
\text { - Comunidades } \\
\text { Autónomas } \\
\text { - I.N.E.M. } \\
\text { - Países de la U.E. } \\
\text { - E.E.U.U. }\end{array}$ \\
\hline & $\begin{array}{c}\text { MODELOS } \\
\text { PARTICULARES }\end{array}$ & $\begin{array}{l}\text { - Centros educativos par- } \\
\text { ticulares } \\
\text { - Equipos sectoriales } \\
\text { - Gabinetes privados de } \\
\text { orientación. } \\
\text { - ... }\end{array}$ \\
\hline
\end{tabular}

Figura 2 Tipología de los modelos de orientación (tomado de Bisquerra y Álvarez, 1998: 57). 


\section{MODELO DE INTERVENCIÓN POR SERVICIOS}

Velaz y Ureta, (1998) resumen las ventajas y limitaciones de este modelo de intervención psicopedagógica (ver figura 3)

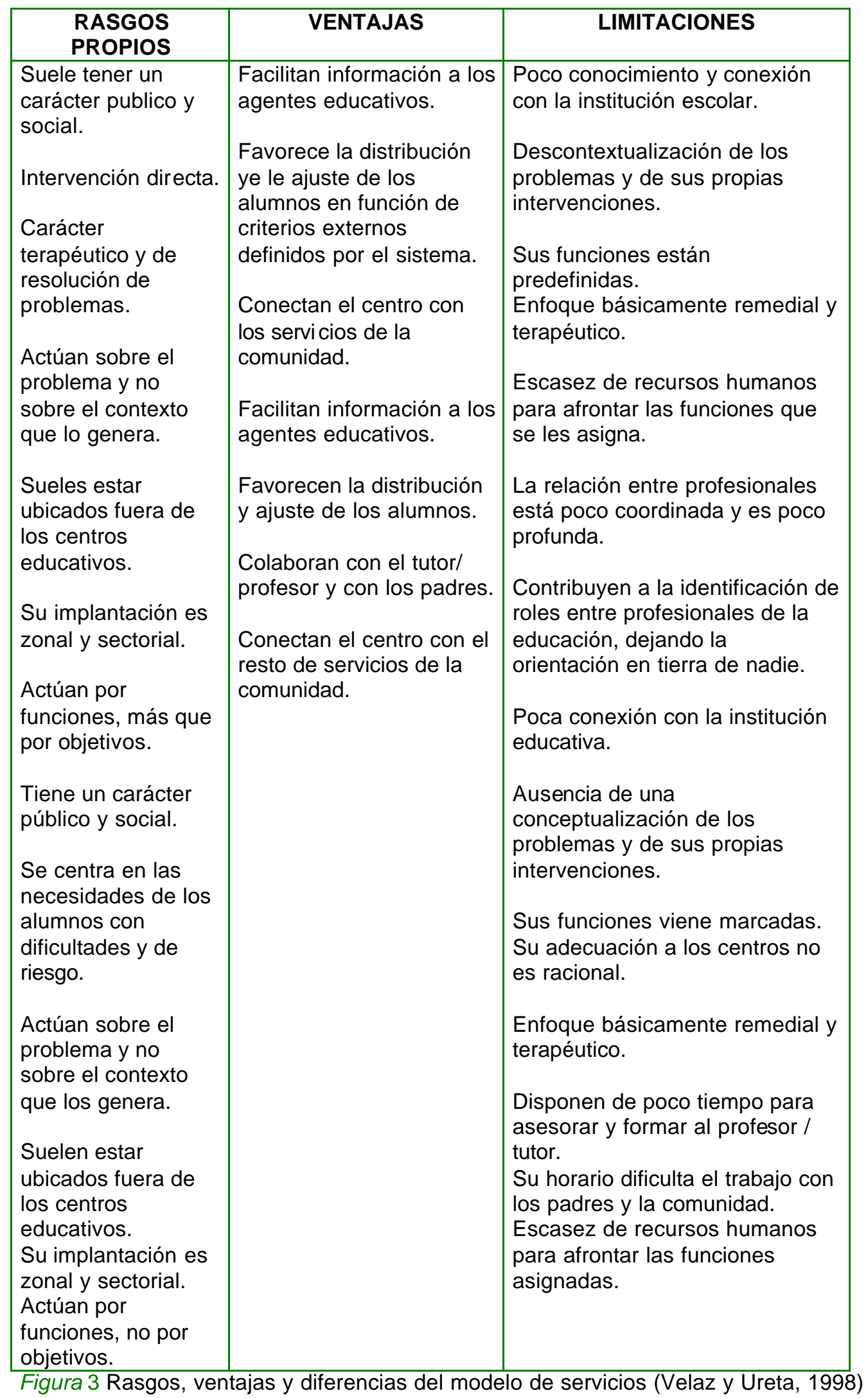


Nos estamos moviendo en un campo caracterizado fundamentalmente por la ambigüedad conceptual, consejo, orientación y psicoterapia (Rodríguez, 1995) y donde tratar de establecer delimitaciones entre ámbitos es bastante complejo como ocurre con el desarrollo personal y orientación psicológica entre guidance y counseling. A esta dificultad en la precisión conceptual contribuye entre otros factores, el contexto en el que nos situemos, americano o europeo.

\section{La orientación en la Unión Europea}

La orientación en la Comunidad Europea a la que se incorpora España en 1986 presenta, en líneas generales las siguientes tendencias (Watts, Dartois y Plant, 1987):

- Carácter continuo de la orientación, tanto profesional como escolar, entendiéndose que debe prolongarse hasta el período de transición a la vida adulta y laboral.

- Por otro lado, la orientación es considerada como la otra cara del proceso educativo. De ahí que se trate de involucrar a todos los agentes educativos en las actividades de orientación, desarrollando para ello formas de apoyarlos en el desempeño de este rol. Esto es, frente a la tendencia de desvitalizar la figura del profesor sustrayéndole de funciones que como educador debe abordar con el grupo-clase, actualmente se defiende la necesidad de ofertar al alumnado una educación integral; para ello se debe propiciar en el profesorado una formación general de amplio espectro, donde las tareas de orientación y tutoría jueguen un papel destacado. También los padres y los agentes sociales deben implicarse activamente en dichas tareas.

- Se defiende un modelo profesional más abierto. Esto es, el objeto de intervención ya no es solo el sujeto aislado, sino los grupos primarios, la institución y la comunidad, dentro de un planteamiento más ecológico y sistémico de trabajo.

- Los objetivos de la intervención no son sólo de índole terapéutica, sino fundamentalmente de prevención y de desarrollo. Esto es, se van a poner las bases para prevenir los problemas en aquellas poblaciones diagnosticadas como de alto riesgo y por otro lado, se van a propiciar las condiciones para promover el desarrollo personal y laboral de los agentes involucrados en el proceso educativo.

- Las estrategias de intervención contemplan la actuación directa sobre el alumnado pero también se enfatiza la importancia de la consulta y formación de los "otros significativos" (padres, profesores y compañeros) así como la actuación sobre los agentes sociales relevantes.

- Los alumnos son considerados como agentes activos en el proceso de orientación. Esto se traduce: a) En el aumento de programas de educación para la carrera (career education), programas de experiencia laboral, etc. diseñados para ofrecer al alumnado una gama de habilidades, actitudes, conocimientos y experiencias que les ayuden a tomar sus propias decisiones. b) Menor énfasis en la testología, para dar paso a un incremento del interés por promover la autoevaluación en lugar de la evaluación del "experto". c) Énfasis en la "educación para la iniciativa" como un modo de desarrollar la autoconfianza y el espíritu de iniciativa (factor fundamental para promover las medidas de autoempleo). 
Salmeron (1996) realiza una revisión de los distintos servicios que se están desarrollando en Europa, resumiendo a continuación sus aportaciones. En las universidades europeas se puede hablar de dos claros y diferenciados tipos de Servicios o Centros de Orientación que no siempre están presentes en la misma Universidad y que, si lo están, generalmente conforman unidades separadas. El primer tipo, el más generalizado, tiene funciones de información y orientación sobre aspectos de carácter vocacional o profesional, en clara conexión con el mundo del trabajo, como es el caso de los Career Services británicos. El segundo tipo, de una orientación psicológica clínica y terapeútica, presta funciones de ayuda individualizada en la resolución de problemas personales. Son los Counseling Center con claros paralelismos con los de las universidades norteamericanas. Finalmente pueden encontrarse oficinas 0 secciones administrativas, más o menos orientadas, que cumplen las funciones de información sobre estudios, o atienden la problemática académica que pueda surgir.

En todos los casos puede hablarse de una clara desconexión con los planteamientos docentes y la dinámica educativa global de la institución.

En cuanto a la financiación, los servicios basados en organizaciones del mercado de trabajo, tienden a estar financiados por el Gobierno Central; los que son del sistema educativo están financiados por el Gobierno Central, las autonomías o las autoridades locales. En definitiva, la mayoría de los servicios son gratuitos para el alumnado.

\section{INGLATERRA}

En el Reino Unido, la orientación y la educación para la carrera se concibe como un proceso que ha de desenvolverse a lo largo de toda la escolaridad, compartida por la institución escolar, la comunidad social y la empresa, adquiriendo un claro protagonismo la simulación de las experiencias laborales. Para Watts (1990) estos programas se proponen los siguientes objetivos:

a) Conocimiento de sí mismo:

b) Conocimiento de las características (habilidades, destrezas, valores e intereses) que definen la clase de persona que es y la clase de persona que quiere llegar a ser;

c) Conocimiento del mundo laboral: conocimiento de las posibilidades que existen, las demandas que hacen y los premios y satisfacciones que puedan ofrecer:

d) Toma de decisiones: adquisición de destrezas que ayudarán a cada uno a tomar decisiones sobre la carrera de la forma más satisfactoria para él mismo;

e) Formación en la transición al empleo: adquisición de destrezas que ayudarán a cada uno a implementar las decisiones de carrera y arreglárselas con las transiciones que conllevan.

Cada universidad, y otras muchas instituciones de enseñanza superior del Reino Unido, cuentan con instituciones creadas para la orientación de sus estudiantes, destacando el sistema tutorial de los Colleges. En algunos casos, estos servicios sirven también a los ex-alumnos de la institución. El resto de las universidades tienen un sistema similar al que presenta Estados Unidos, con funcionamiento independiente de la tarea docente. En la universidad de Cambridge, existen los departamentos de "Career Services" y el "Counselling Services". Los primeros trabajan sobre aspectos de carácter académico, vocacional y profesional con alumnos que están cursando sus estudios, graduados, post-graduados, alumnos que no son miembros de la Universidad de Cambridge y alumnos internacionales. Se destaca la actividad de 
información, especialmente al iniciar la carrera, sobre convalidaciones, becas, e intercambios con otras Universidades. Su misión principal es ayudar en la toma de decisiones. Este servicio te permite contactar con el Careers Adviser (Consejero de carrera), para de una forma más personalizada, recibir una orientación académica o profesional. Existe una clara conexión de este servicio con en mundo del trabajo como lo demuestra su relación con diversos sectores del mundo laboral. El Counselling Service tiene una orientación psicológica clínica y terapéutica, su función principal es la ayuda en la resolución de problemas personales. Este servicio se estableció en 1969 por la creciente demanda y necesidad de los estudiantes. El Counselling Service de la Universidad de Cambridge presta sus servicios tanto a los estudiantes universitarios como a los graduados en problemas como depresión, ansiedad, insomnio, problemas de la alimentación, fobias, etc. Además este servicio te permite contactar con tu tutor o con "The Samaritans" para tener un trato más personalizado.

La Universidad de Londres cuenta con un Counseling Service que al igual que en Cambridge tiene como función primordial la ayuda en la resolución de problemas personales.

Oxford cuenta con un Careers Service, que al igual que en Cambridge te permite contactar con el Careers Advisers para de una forma más personalizada enfocar tu carrera o tu futuro profesional.

En Bristol podemos encontrar el Careers Advisory Service y el Counselling Service con las mismas características antes descritas.

ITALIA

Con respecto a la Orientación Universitaria es el Decreto Ministerial del 21 de julio de 1997, n.245 el que la regula y establece que al inicio de los cursos oficiales se organizarán actividades de orientación en las que se informará a los alumnos sobre la función tutorial y los servicios de orientación con los que cuentan. Por lo tanto, cabe destacar que en todas las Universidades Italianas existe un Servicio de Orientación, dependiente de la propia Universidad y que como norma general ofrecen su ayuda a los alumnos que van a comenzar sus estudios, a los que ya los están realizando, y a los que los han terminado. Su función principal es ofrecer información y asesoramiento sobre aspectos de carácter académico, vocacional y profesional; además de ponerte en contacto con un tutor (Servizio Tutoraro) para de una forma más personalizada analizar los problemas. Otras actividades desarrolladas en estos Servicios son la elaboración de guías interactivas para orientar a los estudiantes, información sobre los distintos campus universitarios, sobre las formas de admisión, asociaciones estudiantiles, cursos, becas, etc.

\section{ALEMANIA}

La Ley alemana de Educación Superior (publicada en enero de 1999) destaca en su sección 14 la función del asesoramiento a los estudiantes: "La institución de enseñanza superior informará a los estudiantes y solicitantes sobre las oportunidades y condiciones de estudio así como del contenido, estructura y requisitos de los cursos.

Durante todo el período académico asistirá a los estudiantes, proporcionándoles consejos sobre la materia. Al final del primer año de estudio, determinará el progresorealizado por los estudiantes, les informará y, si fuese necesario, les aconsejará y orientará". 
La Orientación Universitaria en Alemania consiste en un Servicio de Orientación centralizado y además un grupo de orientadores de los diferentes Departamentos Universitarios, que suelen ser profesores. Su función se centra en información de estudios y orientación profesional, conocimiento del alumno (capacidades e intereses), dificultades de aprendizaje, y problemas personales.

FRANCIA

Desde hace una veintena de años, se han creado servicios de orientación, dentro de cada universidad francesa, que se encargan de los problemas de acogida, orientación e inserción profesional de los estudiantes: se trata de los SCUIO. Sus misiones son muy amplias, los problemas de orientación de los estudiantes a los que se supone que deben responder aumentan vertiginosamente. La calidad de estas intervenciones se debe a que de hecho se basan generalmente en investigaciones llevadas a cabo desde hace años en Norteamérica en el campo del desarrollo de carrera.

La problemática de la orientación en las universidades francesas podría resumirse como sigue: por un lado, hay una necesidad de aportar respuestas de calidad a un gran número de estudiantes; por otro lado falta personal especializado, a pesar de que posean en general los presupuestos teóricos y educativos para tratar el problema, y por último está la dificultad para movilizar el inmenso recurso cuantitativo y cualitativo que representan los profesores.

La Universidad Claude Bernard de Lyon es la primera que ha implantado en Francia un dispositivo que afecta a 2.000 estudiantes del primer año. Se trata de un módulo obligatorio que concierne a todos los recién llegados que se nscriben en el primer año del DEUG de ciencias, ya sea por elección o por asignación. Dentro de este contexto, se les conduce a realizar un proceso de exploración de los medios profesionales, algo que no es habitual en la universidad. La originalidad también reside en que están enmarcados en pequeños grupos de veinte o veinticinco estudiantes y acompañados por un profesorinvestigador, lo que representa alrededor de setenta de estos últimos para 2.000 estudiantes. El papel que desempeñan los profesores en estos pequeños grupos es conducir a los estudiantes a la metodología de la investigación para que la apliquen a un objeto particular: la exploración de un campo profesional que les interese verdaderamente, aunque no esté relacionado forzosamente con los estudios iniciados. En esta enseñanza se intenta implicar, involucrar al máximo al estudiante, partir de él mismo, de sus intereses, de lo que puede vibrar en él siempre en relación con el mundo laboral.

\section{Servicios de orientación en las universidades españolas}

En nuestro contexto destacamos el Centro de Apoyo al Estudiante (CAE) de la Universidad de Alicante. Cuenta con un equipo de psicólogos y trabajadores sociales que vienen atendiendo a los estudiantes de la Universidad de Alicante que ven afectado su rendimiento académico por motivos como; Falta de concentración, problemas de memoria y bajo rendimiento, así como dificultades de adaptación a la vida universitaria, conflictos con el profesorado o miedo a los exámenes. Entre las condiciones que imponen para la prestación de estos servicios se consideran; (i) ser estudiante de la Universidad de Alicante y demostrarlo mediante acreditación, (ii) se respetará el horario acordado en la cita, (iii) la atención no podrá extenderse más allá de tres citas con el solicitante por el mismo motivo de consulta y (v) ni los técnicos que prestan el servicio ni la propia Universidad se hacen responsables de las decisiones adoptadas por los solicitantes tras la prestación del servicio. 
La Universidad de Granada, a través del Vicerrectorado de Estudiantes consideró prestar atención y asesoramiento directo a los alumnos matriculados en los distintos estudios universitarios, con el objetivo de atender las necesidades que presenten dichos alumnos. Así como las de su integración y adaptación en el medio universitario desde los principios de derecho a la igualdad de oportunidades, a la participación y a la plena inserción y transición a la vida laboral. Con este propósito nace en 1998 el Gabinete Psicopedagógico de Orientación de la Universidad de Granada. Desde este servicio se intentan arbitrar aquellas soluciones que se consideran más adecuadas y sean capaces de satisfacer las necesidades, disfunciones, interés... del alumnado durante el periodo de tiempo que permanezcan en la universidad. Pretende ser un servicio de orientación a la comunidad que mejore las condiciones en que los estudiantes acceden a estudios universitarios y desarrollen la carrera en las condiciones más óptimas a la vez que le ayuden a integrarse mejor en los contextos sociales que se relacionan con su vida (Salmerón, Pérez y Peñafiel, 2000).

En el campus de Cuenca de la Universidad de Castilla la Mancha propone el Servicio de orientación de Cuenca (SOCU) cuyos objetivos destacamos (Rincón, Bayot, García y Argudo, 2000); (i) Situar al estudiante en el entorno universitario general y en el propio de su falta o escuela universitaria donde inicie sus estudios, (ii) ayudar a conocer los inerarios formativos de grado y postgrado de las distintas titulaciones, (iii) apoyar los procesos de aprendizaje en la universidad, (iv) orientar las tomas de decisiones en el mundo laboral, (v) implicar progresivamente en el servicio a estudiantes y profesores de las distintas titulaciones.

Tal y como se especificaba en el proyecto inicial, las actuaciones a desarrollar se han distribuido en los programas o apartados que enumeramos: (a) Acogida e incorporación al contexto universitario: ubicación y conocimiento del entorno; asamblea general y presentación del SOUC, (b) Orientación personal; prevención y desarrollo, (c) Orientación académica; el estudio en la universidad, estrategias y planificación del tiempo, base de datos con las características de las asignaturas de las distintas carreras; planes de estudios, itinerarios y optatividad. Y (d) Orientación profesional; recogida de información de los egresados en promociones anteriores de algunas carreras del campus de Cuenca.

Centrándonos en orientación personal; prevención y desarrollo, se han programado jornadas sobre "hábitos de estudio y relajación" con el objetivo de facilitar a los estudiantes habilidades y destrezas para enfrentarse al periodo de exámenes de febrero. Además, está previsto realizar un seminario sobre el SIDA, durante el primer trimestre del curso próximo y otras actividades relacionadas con las drogodependencias.

El equipo de SOUC apenas ha iniciado sus andadura y no ha tenido tiempo de evaluar el resultado de sus intervenciones. Sin embargo, ya contamos con algunos indicadores que nos permiten valorar la experiencia positivamente; (i) La opiniones de los estudiantes en relación a la necesidad de un servicio de orientación. Y (ii) Acogida de las actividades propuestas hasta ahora; sesiones de preparación para los exámenes, asesoramiento a los alumnos primero.

Programa de Aconsejamiento Psicológico en la Facultad de Medicina de la Universidad de Barcelona, al igual que otros centros docentes, éste centro se ha planteado la necesidad de ofrecer ayuda a las dificultades emocionales de los estudiantes. Este proyecto presta apoyo psicológico teniendo presente las situaciones específicas de la Facultad de Medicina. A grandes rasgos esta especificidad se concreta en la necesidad de afrontar; (i) un primera etapa preclínica que exige al alumnado demandas de control emocional y eficacia en el rendimiento académico, marcado por una notable competencia y (ii) una última etapa, marcada por la incertidumbre de su integración laboral, mediatizada por el examen MIR. 
El Servicio de Orientación Educativa y Psicológica (SOEP) del SAPE de la Universidad de A Coruña. Esta constituido por dos orientadores profesionales en los campos de la evaluación, diagnóstico, formación, intervención y orientación de la psicopedagogía y la psicología. Se divide en; orientación psicológica dirigido por un psicólogo y en orientación académica / educativa dirigida por una psicopedagoga. La asistencia se realiza a través de consultas individuales en el SAPE (Campus da Zapateira y Casa do Francés), que los interesados habrán solicitado previamente. También además de las consultas de orientación y asistencia individual, el SOEP, ofrece la posibilidad de realizar cursos de formación al estudiante en modalidad presencial y/o virtual, con el objeto de capacitarlos en las habilidades necesarias para la superación de las posibles necesidades que se le pueden presentar en el área académica y/o psicológica. Se ofrece además un servicio de autoorientación virtual.

SOUCAN, es un servicio de la Universidad de Cantabria, que viene ofreciendo sus servicios desde 1996, año en que se impulsó, a través del primer convenio de colaboración entre la Universidad de Cantabria y el Gobierno de Cantabria.

Entre sus principales objetivos podemos señalar; (i) Proporcionar información y el apoyo necesario para que los alumnos tomen las decisiones adecuadas en el campo de su orientación personal, académica y profesional, (ii) coordinar todos los recursos que permitan la consecución de este fin, tanto dentro de la Universidad de Cantabria, como en el resto de la Comunidad Educativa, (iii) implicar a los profesores universitarios en tareas de orientación y asesoramiento académico como parte integrante de la tarea docente, y (v) evaluar y mejorar la calidad de todas sus actividades de orientación.

SOUCAN, con la intención de adaptarse constantemente a las necesidades de los usuarios (alumnos, profesorado de secundaría o de la Universidad de Cantabria, Orientadores ...) ha ido evolucionado en múltiples aspectos, tales como funcionamiento interno y externo, contenido, organización y ampliación de sus actividades, aunque siempre ha conversado su esencia respecto al marco conceptual del que fue su origen: Un sistema que englobe diferentes acciones de orientación, información, coordinación de recursos y normalización para apoyar al alumnado en su proceso de formación personal, académico y profesional, en las tres etapas; preuniversitaria, universitaria y postuniversitaria.

Dispone de un servicio de Apoyo Psicológico, cuya finalidad es ofrecer solución a determinadas dificultades y / o trastornos psicológicos y / o educativos que necesitan de un apoyo más permanente y especializado. Así, en general, se ofrece al alumnado desde una orientación personalizada que se realiza durante varias sesiones, hasta un tratamiento terapéutico, desarrollado por un servicio externo al propio servicio, en donde varios profesionales especializados en diferentes temas abordan el tratamiento del alumno.

Actualmente se están poniendo en marcha diversos servicios de orientación del departamento de orientación en la Universidad de Extremadura propuestas por Sánchez, Pajuelo, Vicente y Sosa (2000).

\section{REFERENCIAS BIBLIOGRÁFICAS}

ÁlvareZ GonZÁleZ, M. y BISQUeRRA AlZINA, R. (1997). Los modelos de orientación e intervención psicopedagógica, en AIDIPE (comp..) (22-25): Actas del VIII Congreso Nacional de Modelos de Investigación Educativa, Sevilla, Universidad de Sevilla.

BISQUeRRA ALZINA, R. (1992). Proyecto docente e investigador. Universidad de Barcelona. 
BISQUERRA ALZINA, R. (1998). Modelos de Orientación e Intervención psicopedagógica. Barcelona: Editorial Praxis.

BISQUERRA, R. y ÁlvAREZ, M. (1996). Modelos de intervención en orientación, en Bisquerra, R. y Álvarez, M. (coords.) (331-351). Manual de orientación y tutoría. Barcelona: Praxis.

CAstellano, F.(1995). La orientación educativa en la Universidad de Granada. Granada: Servicio de Publicaciones de la Universidad de Granada.

MIRAS, M. (1986). La intervención psicopedagógica. Cuadernos de Pedagogía, 139, 79 - 82.

RepPetto TalaVera, E.(1994). Programa de exploración y planificación de la carrera y el empleo (E.P.C.P.).

Reppetto talavera, E., Rus Anega, V. y Balaguer, J.(1995). Orientación Educativa e Intervención Psicopedagógica. Educación Permanente. Madrid: Universidad Nacional de Educación a Distancia.

RIVAS, F. y otros (1998). SAV. Sistema de ayuda vocacional. Valencia: Consejería de Cultura, Educación y Ciencia.

RODRíGUEZ DieGUEZ, A.(1990). Aproximación a la educación vocacional”. Una perspectiva desde la reforma educativa, 8, $125-143$.

Rodríguez Espinar, S. (coord..), ÁlvareZ, M., Echeverría, B. y Marín, M.A.(1993). Teoría y práctica de la orientación educativa. Barcelona: PPU.

RODRíGUEZ MORENO, Mª L. L.(1995). Educación para la carrera y diseño curricular. Barcelona: Universitat de Barcelona.

Sánchez Herrera, S., Pajuelo Morán, C., Vicente Castro, F. y Sosa Baltasar, D..M. (2000). Propuesta de implementación de un departamento de orientación en la Universidad de Extremadura, en Salmeron, V. y López, V.L.(321 - 325): Orientación Educativa en las Universidades. Granada: Grupo Editorial Universitario.

SALmerón PÉREZ, H.(1996). Los servicios de orientación en la Universidad; Procesos de creación y desarrollo. Informe para la UNESCO de la Comisión internacional sobre Educación para el siglo $\mathrm{XXI}$.

SANTANA VEGA, L.E. y SANTANA BONILLA, P.(1998). El modelo de consulta / asesoramiento en orientación. Revista de Investigación Educativo, 16, 2, 59 - 77.

SobRADO, L. (1999). Servicios internos de orientación en el Sistema Educativo Español. Revista de Orientación y Psicopedagogía, 17, 207 - 226.

VÉLAZ de C. y URETA, M.(1998). Orientación e intervención psicopedagógica. Ediciones Aljibe: Granada.

WATTS, A. G.(1990). The Role of Guidance in Educational Change. En Watts, A. G. (eds.) (10-13): Guidance and educational change: a cross - sectorial eview of policy and practice. CRAC Pub., Hobsons Pub., Cambridge.

WATTS, A.G., DARTOIS, C. y PLANT, P. (1987). Career guidance service within the European Community: Constrast and common trends. International Journal for de Advancement of Counselling. 


\title{
Contactar
}

Revista lberoamericana de Educación

\author{
Principal OEI
}

